5-9-2006

\title{
Single-Molecule Transistors: Electron Transfer in the Solid State
}

\author{
Douglas Natelson
}

Lam H. Yu

Jacob W. Ciszek

Loyola University Chicago, jciszek@luc.edu

Zachary K. Keane

James M. Tour

Follow this and additional works at: https://ecommons.luc.edu/chemistry_facpubs

Part of the Chemistry Commons

\section{Recommended Citation}

Natelson, D, LM Yu, JW Ciszek, ZK Keane, and JM Tour. "Single-molecule transistors: electron transfer in the solid state" in Chemical Physics 324(1), 2006.

This Article is brought to you for free and open access by the Faculty Publications and Other Works by Department at Loyola eCommons. It has been accepted for inclusion in Chemistry: Faculty Publications and Other Works by an authorized administrator of Loyola eCommons. For more information, please contact ecommons@luc.edu. cc) (i) $\ominus$

This work is licensed under a Creative Commons Attribution-Noncommercial-No Derivative Works 3.0 License. (c) 2005 Elsevier BV. 


\title{
Single-molecule transistors: Electron transfer in the solid state
}

\author{
Douglas Natelson ${ }^{\mathrm{a}, *}$, Lam H. Yu ${ }^{\mathrm{a}}$, Jacob W. Ciszek ${ }^{\mathrm{b}}$, \\ Zachary K. Keane ${ }^{\text {a }}$, James M. Tour ${ }^{\text {b,* }}$ \\ ${ }^{a}$ Department of Physics and Astronomy, Center for Nanoscale Science and Technology, Rice University, 6100 Main Street, Houston, TX 77005-1892, USA \\ ${ }^{\mathrm{b}}$ Department of Chemistry, Center for Nanoscale Science and Technology, Rice University, 6100 Main Street, Houston, TX 77005-1892, USA
}

Received 28 June 2005; accepted 25 November 2005

Available online 18 January 2006

\begin{abstract}
Single-molecule transistors (SMTs) incorporating individual small molecules are unique tools for examining the fundamental physics and chemistry of electronic transport in molecular systems at the single nanometer scale. We describe the fabrication and characterization of such devices, and the synthesis and surface attachment chemistry of novel transition metal complexes that have been incorporated into such SMTs. We present gate-modulated inelastic electron tunneling vibrational spectroscopy of single molecules, strong Kondo physics $\left(T_{\mathrm{K}} \sim 75 \mathrm{~K}\right)$ as evidence of excellent molecule/electrode electronic coupling, and a demonstration that covalent attachment chemistry can produce SMTs that survive repeated thermal cycling to room temperature. We conclude with a look ahead at the prospects for these nanoscale systems.
\end{abstract}

(C) 2005 Elsevier B.V. All rights reserved.

Keywords: Single-molecule transistors; Kondo physics; Molecular electronics

\section{Introduction}

Electron transfer through molecules has been a topic of intense study for decades. Only in recent years, however, techniques have been developed to experimentally access the electronic conduction properties of individual small molecules. Even restricting oneself to small molecules, the variety of systems available for examination is enormous, from saturated alkanes and conjugated oligomers to fullerenes and organometallic complexes. Combined with the ability to vary attachment chemistry and derivatives within a family of compounds, electrodes spaced at the nanometer scale make possible systematic studies of electron transport in the single-molecule limit.

The scanning tunneling microscope (STM) is an example of such an enabling technology for single-molecule studies. In such a device, molecules of interest are physi-

\footnotetext{
* Corresponding authors. Tel.: +1 713348 3214; fax: +1 7133484150 (D. Natelson), Tel.: +1 713348 6246; fax: +1 7133486250 (J.M. Tour)

E-mail addresses: natelson@rice.edu (D. Natelson), tour@rice.edu (J.M. Tour).
}

sorbed or chemisorbed onto a smooth conductive substrate, while a metal tip is scanned over the surface, with tip height controlled by a feedback loop that maintains a constant tunneling current between the tip and the substrate. As a result, the apparent height of molecular features in STM is a convolution of the topography and local electronic properties. In a highly stable STM, at a desired location the feedback loop may be turned off, and a current/voltage characteristic may be acquired as a function of tip/substrate bias. Assuming that the electronic density of states of the tip is relatively featureless, this technique, known as scanning tunneling spectroscopy (STS), allows the measurement of the local density of states induced by the molecule. This novel spectroscopy has been revelatory, allowing the solid state observation of the alignment and broadening of occupied molecular orbitals [1], vibronic effects [2], inelastic electron tunneling via molecular vibrations [3] and spin flips [4], and electronic correlations such as Kondo physics [5].

While these two-terminal measurements are very powerful, they lack the ability to tune the energies of the molecule's electronic states independent of the applied bias. 
STM in the presence of electrolytic solutions is a recent innovation that allows in situ tuning of molecular redox state combined with STS. However, such electrochemical "gating" is restricted to operations near room temperature. Indeed, the exponential dependence of tunneling conductance on interelectrode distance coupled with the strong temperature response of piezoelectric actuators makes performing STS at fixed junction configuration over a broad temperature range extremely challenging.

In this paper, we report recent single-molecule electron transport experiments in planar nanoscale junctions capacitively coupled to gate electrodes. These single-molecule transistors (SMTs), developed quite recently [6], allow studies of electronic transport in individual small molecules from room temperature to cryogenic temperatures, with gate modulation of the charge state of the molecule [713]. Below we describe sample fabrication and characterization, with an emphasis on the importance of a statistical approach with large numbers of candidate devices and control experiments. We discuss the synthesis methods used to produce the novel transition metal coordination complexes that we study, and the thiocyanate-based self-assembly mechanism used to attach the conjugated ligands to our metal source and drain electrodes. After summarizing the conductance mechanisms at work in such devices, we present data showing inelastic electron tunneling in single molecules via vibrational states; strong Kondo effects in single molecules that confirm the extremely efficient molecule/ metal electronic coupling possible with appropriate ligands and attachment chemistry; and finally evidence that such SMTs can survive multiple thermal cycling between cryogenic conditions and room temperature. We conclude with a discussion of open scientific issues and future directions for this exciting, nascent field of research.

\section{Fabrication and characterization}

We use an electromigration procedure [14] to fabricate single-molecule transistors. Thin $(1 \mathrm{~nm} \mathrm{Ti} / 15 \mathrm{~nm} \mathrm{Au})$ metal constrictions are defined by electron beam lithography and e-beam evaporation. The choice of electrode materials is one of convenience: gold surface chemistry is well known and gold is robust against the formation of a stable oxide; the titanium serves as an adhesion layer. For our experiments, the substrate is a degenerately doped $\mathrm{p}+$ silicon wafer coated with $200 \mathrm{~nm}$ of thermally grown $\mathrm{SiO}_{2}$. The substrate itself is used as a gate. Following evaporation and liftoff, the chip is cleaned in an oxygen plasma for $1 \mathrm{~min}$, and molecules of interest are deposited. The molecules used in this work deposit by self-assembly during $48 \mathrm{~h}$ of immersion in a $2 \mathrm{mM}$ solution in tetrahydrofuran (THF) in the dark.

When assembly is complete, the substrate, typically patterned with at least 45 constrictions, is placed into a variable temperature vacuum probe station and pumped to high vacuum conditions. Electromigration is performed at room temperature to "pre-break" constrictions to a resistance of a few hundred Ohms. The sample is then cooled to cryogenic temperatures $(\sim 5 \mathrm{~K})$ and further electromigration is performed to break the sample to a resistance greater than $20 \mathrm{k} \Omega$. The hope is that some fraction of the resulting interelectrode gaps will be bridged by single molecules, and that those molecules will have significant capacitive coupling to the underlying gate to permit transistor action. This final procedure is performed in the cryopumped ultrahigh vacuum environment to minimize the risk of surface contamination by unintended adsorbates (see Fig. 1).

As has been discussed elsewhere [11], this fabrication procedure is inherently statistical. Not every interelectrode gap will contain a molecule. The precise morphology of each interelectrode gap and electrode surface is unique and unknown. The details of metal/molecule bonding (orientation, binding sites) are unknown. In this respect, SMTs are far inferior to STM approaches that allow significant surface characterization. Given the exponential dependence of tunneling conductance with distance, one might well imagine that every device would have properties completely different from every other device, and that no general conclusions could be drawn. Coupled with the lack of microscopy tools that can directly examine the interelectrode gap region, the situation may appear bleak.

Fortunately, however, an appropriate statistical approach with systematic control experiments enables real progress to be made in spite of these complications. In fact, the exponential sensitivity of tunneling to geometry works in favor of the experimentalist, for precisely the same reason that STM tips do not have to be prepared with atomic precision. The interelectrode conductance is dominated by the subnanometer-scale region of closest interelectrode separation. The result is that the gap location through which most of the current flows is most likely to contain zero or one molecule, rather than dozens.

Device characterization is performed by measuring the drain current $\left(I_{\mathrm{D}}\right)$ as a function of source/drain voltage $\left(V_{\mathrm{SD}}\right)$ and gate voltage $\left(V_{\mathrm{G}}\right)$ at low temperatures. More than $80 \%$ of the initial constrictions are typically found to still exhibit some measurable interelectrode conduction at low temperatures following the completion of the
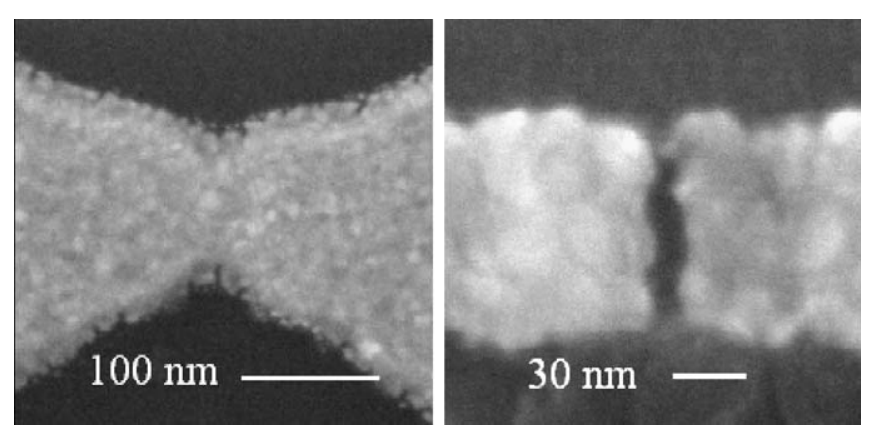

Fig. 1. Metal nanoconstriction before (left) and after (right) electromigration procedure used to break it into distinct source and drain electrodes separated at the nanometer scale. 
electromigration process. Upon subsequent scanning electron microscope (SEM) imaging, the nonmeasurable electrode pairs are typically observed to have interelectrode separations $5 \mathrm{~nm}$ in size or greater. For the measurable electrode sets, the results have been found to fit into three broad categories. Weakly nonlinear $I_{\mathrm{D}}-V_{\mathrm{D}}$ curves with no gate dependence account for half of the remaining devices, and are assumed to be vacuum gaps. Roughly $25 \%$ of the original electrodes exhibit significantly nonlinear $I_{\mathrm{D}}-V_{\mathrm{D}}$ curves with no gate dependence. Without any gate modulation, it is generally not possible to tell whether such data are due to molecules, adsorbed contaminants, or metal nanoparticles produced during the electromigration process. We do not study this class of devices. The remaining $15 \%$ of the starting devices exhibit significant $I_{\mathrm{D}}-V_{\mathrm{D}}$ nonlinearities with good gate modulation. These are the devices of interest. Remarkably, these rough proportions of $I_{\mathrm{D}}-V_{\mathrm{D}}$ properties are robust across many different molecules, and even across research groups.

In control experiments with no molecules, approximately $1-2 \%$ of the total devices are found to exhibit gate response. On the basis of their conductance properties (see below), these are judged to be unintentionally produced metal nanoparticles. Such particles can be produced deliberately for study, if desired, and have been reported by others in similar electromigrated systems [15].

Generally the best control experiment to run is a comparison of significant numbers of devices fabricated with the molecule of interest, and similar quantities of devices made incorporating either control molecules (e.g., alkane chains) or just the solvent in question. Discriminating between metal nanoparticles and actual molecules is discussed in more detail below.

When considering potential improvements on this experimental approach, a desirable variation becomes clear. It would be best to take a constriction-bearing substrate, clean it in UHV using standard surface science techniques, perform the electromigration process in UHV, and sublime a dilute coverage of molecules onto preexisting, characterized interelectrode gaps. Resulting devices could then be measured in situ. Something similar to this, though with a different junction fabrication method, has been attempted elsewhere [9]. The main reasons that such an approach has not been implemented are high cost, because of the complexity of the resulting UHV chamber/measurement system, and the challenge of volatilizing candidate molecules.

\section{Properties and attachment of the transition metal complex}

The transition metal complex consists of a cobalt atom in a $2+$ oxidation state surrounded by two anionic ligands for an overall neutral molecule with no counter ions. The oxidation of the metal center from the $2+$ state to the $3+$ state is the only transition observed when scanned from -1 to $0.5 \mathrm{~V}$ (vs. $\mathrm{Ag} \mid \mathrm{AgNO}_{3}$ ). The respective reduction and oxidation occur at -0.012 and $0.144 \mathrm{~V}$ at a $20 \mathrm{mV} / \mathrm{s}$ scan rate. Reduction of the ligand is possible at sufficiently negative potentials $(\sim-2 \mathrm{~V})$ with the SCN moiety being the most probable location. The ligand provides a compressed octahedral environment (Fig. 2a) and a high spin (3/2) system as confirmed by SQUID and EPR spectroscopy. Orbital energy levels are consistent with that of compressed octahedral geometry $\left(E \mathrm{~d}_{x y}<\mathrm{d}_{y z}, \mathrm{~d}_{x z}<\mathrm{d}_{x^{2}-y^{2}}<\mathrm{d}_{z^{2}}\right)$. Hence the unpaired electrons are expected to occupy the $\mathrm{d}_{z^{2}}$, $\mathrm{d}_{x^{2}-y^{2}}$, and $\mathrm{d}_{x z}$ or $\mathrm{d}_{y z}$ with the degeneracy of the latter two being lifted by Jahn-Teller distortion.

This molecular system is designed for simplistic assembly and takes advantage of the relatively new process of thiocyanate assembly. Thiocyanates are converted by the surface via cleavage of the $\mathrm{S}-\mathrm{CN}$ bond to the same thiolate species found in the ubiquitous thiol or disulfide assemblies. The resultant adsorbed $\mathrm{CN}$ leaves the surfaces as $\left[\mathrm{Au}(\mathrm{CN})_{2}\right]^{-}$. Hence exposure of the metal complex to a gold surface will give the same bonding motif as if starting with the free thiol (Fig. 2b). Aromatic free thiols are troublesome, easily giving disulfide polymers when dealing with $\alpha, \omega$-dithiols, a problem adverted by use of the thiocyanate.

Assembly on gold gives a thickness consistent with what is expected for a loosely packed layer of bulky molecules. Other surface characterizations (XPS, surface IR) show species pre- and post-assembly that are virtually identical except for the two thiocyanates that have been converted
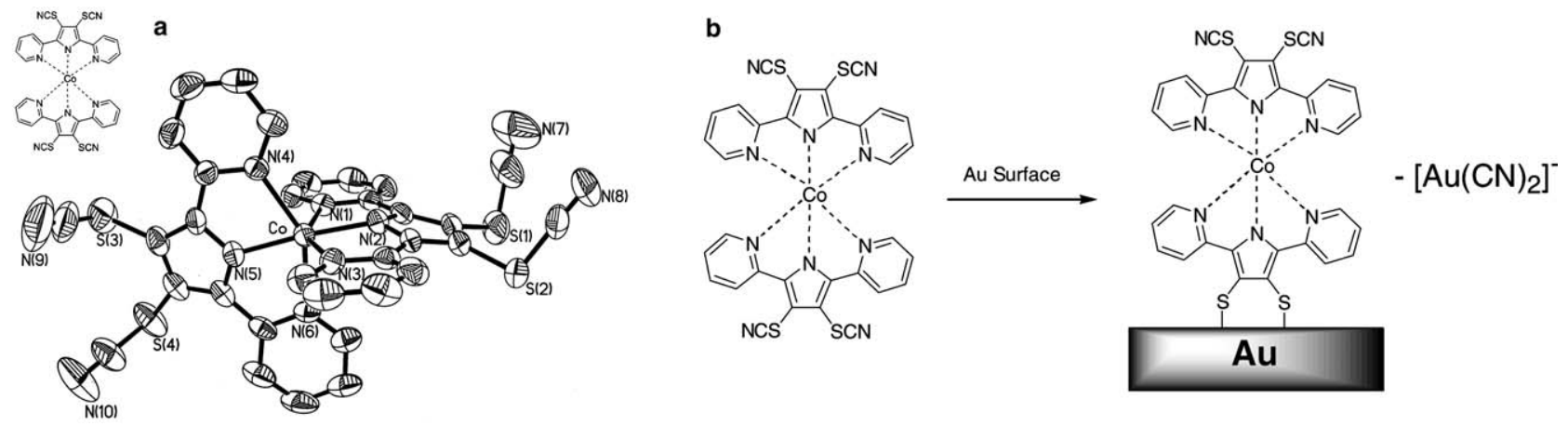

Fig. 2. Visualization of the cobalt complex and its assembly. (a) ORTEP of the cobalt complex from X-ray crystallography. (b) The assembly sequence on gold and final assembled structure. 
to thiolates during assembly. This confirms that minimal change occurs in the molecule during assembly.

\section{Conduction processes and single-electron transistors}

For reasons that are not entirely clear, the SMTs that have so far been examined are observed to function as single-electron transistors (SETs) [16]. A generic SET is shown in Fig. 3a, and consists of an "island" in tunneling contact with source and drain electrodes, and capacitively coupled to a gate. Such systems have been studied extensively using patterned metal islands [16], gate-defined "quantum dots" in semiconductor heterostructures, and nanoparticle islands [17].

These devices are commonly called "single-electron" transistors because the conductance through such a device can be modulated dramatically by changing the average charge on the island by a single electron. Fig. 3c maps the differential conductance, $\partial I_{\mathrm{D}} / \partial V_{\mathrm{SD}}$, of a generic SET as a function of source-drain bias, $V_{\mathrm{SD}}$, and gate voltage, $V_{\mathrm{G}}$. Fig. 3b shows the energetics of such a system, and Fig. 3d enumerates possible electronic transport mechanisms.

As is shown in Fig. 3b, the spectrum of allowed singleparticle states of the island is discrete, while the source and drain electrodes can generally be treated as having a continuum of states filled up to some Fermi energy (chemical potential). There is a highest occupied island state (in a molecule, this would be the highest occupied molecular orbital, HOMO), and a lowest unoccupied state (in a molecule, the lowest unoccupied molecular orbital, LUMO). The energetic separation between island single-particle states consists of two components. The first is the single-

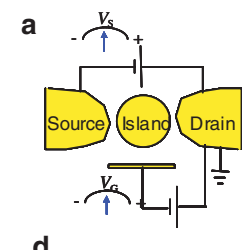

d
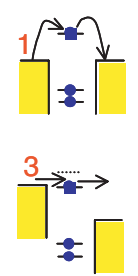

b
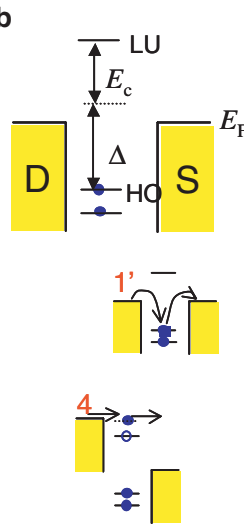

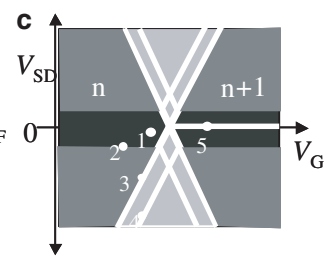

2
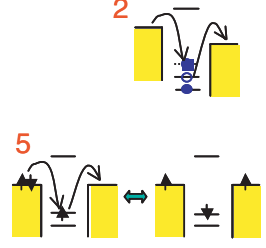

Fig. 3. Conduction in single-electron transistors. (a) Cartoon of generic SET. (b) Energy level diagram of generic SET neglecting spin, showing highest occupied (HO) and lowest unoccupied (LU) single-particle states of the island. (c) Differential conductance (white $=$ high $\partial I_{\mathrm{D}} / \partial V_{\mathrm{SD}}$, black $=$ low $\left.\partial I_{\mathrm{D}} / \partial V_{\mathrm{SD}}\right)$ map of a generic SET as a function of source/ drain and gate voltages, showing a single charge transition point. To the left of the transition, the island has on average $n$ electrons, while to the right of the transition the average island electron number is $n+1$. (d) Specific conduction processes corresponding to the numbered locations in $(c)$. particle level spacing, $\Delta$, analogous to the splitting between states in a "particle-in-a-box" picture. In the absence of electron-electron interactions, this is the minimum energy required to form an electron-hole excitation on the island. The second contribution is the Coulomb charging energy, $E_{\mathrm{c}}$. This charging energy is a zeroth order means of dealing with electron-electron interactions. In the absence of more subtle issues (e.g., spin and exchange), the total energy required to add an additional electron to the island is $\Delta+E_{\mathrm{c}}$. The charging energy is usually written in terms of a classical capacitance, though the physical interpretation of this in molecular structures is subtle.

The capacitive coupling to the gate allows the discrete spectrum of the island to be shifted energetically relative to the chemical potential of the source and drain. The result is that the gate can modulate the equilibrium charge state of the island. In molecular parlance, the gate allows tuning through redox transitions of the island.

It is important to note that $V_{\mathrm{G}}$ does not correspond directly with the oxidation/reduction potentials encountered in electrochemical experiments. Rather, there is a sample geometry dependent constant of proportionality that converts $V_{\mathrm{G}}$ into actual shifts in island energy. This constant may be inferred from the slopes of the diagonal lines in Fig. 3c. If every device were precisely identical (that is, the same molecular orientation bonded the same way onto the same crystallographic surfaces of the metal electrodes, with identical charge environments and surface states around the metal), then the locations of conductance features as a function of $V_{\mathrm{G}}$ would reproduce perfectly from device to device, and would correlate with electrochemical measurements. However, since molecule-metal charge transfer (which defines the relative energies of the HOMO/LUMO and the metal chemical potentials) and the local charge environment (via trap states in the oxide, for example) can vary strongly from device to device, significant variation is seen in the $V_{\mathrm{G}}$ location of conductance features. We note that such "offset charge" variations are routinely seen in metal and semiconductor single-electron devices.

For low biases $\left(1,1^{\prime}\right)$ the average number of electrons on the island is fixed; the chemical potential of the source and drain lies within a gap in the island spectrum, and transport is suppressed at $T=0$. Historically this suppression is called Coulomb blockade because in SETs with larger islands, $E_{\mathrm{c}} \gg \Delta$. The requirement for observing robust Coulomb blockade is $\Delta+E_{\mathrm{c}} \gg k_{\mathrm{B}} T$. In the blockaded configuration, transport can only occur by higher order tunnelling through virtual states. An example of this is "superexchange" [18], and in SETs such processes are called "elastic cotunnelling" [19]. At higher biases in the blockaded regime, "inelastic cotunnelling" via an excited virtual state [2] is possible. For an excitation of energy $E^{*}$, the opening of the inelastic channel results in a feature in $\partial^{2} I_{\mathrm{D}} / \partial V_{\mathrm{SD}}^{2}$ at $e\left|V_{\mathrm{SD}}\right|=E^{*}$. Inelastic cotunnelling via electronically excited states has been seen in semiconductor [20] and carbon nanotube SETs [21]. Inelastic cotunnelling via 
vibrationally excited molecules is responsible for conventional IETS [22], but has never been studied in three-terminal devices until now. IETS lineshapes are predicted to vary significantly depending on the energetics of the virtual states [23], and can be peaks, dips, or intermediate structures in $\partial^{2} I_{\mathrm{D}} / \partial V_{\mathrm{SD}}^{2}$. At still higher source-drain biases [3] Coulomb blockade is lifted leading to significant resonant conduction, while at still higher biases [4] additional resonant conduction occurs when $e V_{\mathrm{SD}}$ is sufficient to leave the island in an electronically [24-26] or vibrationally $[6,7,10]$ excited state.

One additional conduction mechanism is the Kondo resonance. If there is an unpaired spin localized to the island, virtual processes like the one shown in Fig. 3c [5] can occur. The result is antiferromagnetic exchange between the conduction electrons of the leads and the spin localized to the island. Below a characteristic temperature $T_{\mathrm{K}}$ that depends exponentially on the matrix element coupling the spin and the conduction electrons [27,28], resonant transmission from the source to the drain is possible. The signature of this mechanism is the appearance of a maximum in the differential conductance at zero bias (the thick white line at $\left.V_{\mathrm{SD}}=0\right)$ for $T<\sim T_{\mathrm{K}}$. The width of this resonance is $\sim 2 k_{\mathrm{B}} T_{\mathrm{K}} / e$, and for an island with symmetric coupling to source and drain, the peak conductance for $T \ll T_{\mathrm{K}}$ saturates to $2 e^{2} / h$, the maximum possible conductance for a single channel. Kondo resonant charge transfer has been observed in semiconductor quantum dots at sub-Kelvin temperatures [29-31], and in SMTs at higher temperatures $[7,8]$.

When one of the island states is shifted, via a gate potential, to be degenerate with the chemical potential of the source and drain, blockade is lifted and source/drain current may flow at essentially zero source/drain bias. Such a point is called a charge transition or charge degeneracy point, because the island is on the cusp of changing its average charge. In single-molecule transistors, $\Delta$ and $E_{\mathrm{c}}$ are typically hundreds of $\mathrm{meV}$. This is in strong contrast to metal or gated semiconductor quantum dots, where energy scales are hundreds of times lower. As a result, the effects described above are in principle observable at room temperature in SMTs.

These high energy scales, together with fundamental chemical stability considerations, lead to a further control/diagnostic, as alluded to in Section 2. Electrochemical measurements (e.g., cyclic voltammetry) give an upper limit on the number of redox changes that may reasonably be expected in a SMT. The gate coupling in a SMT is undoubtedly far less efficient at compensating molecular charge than the screening ions in solution Angstroms away in the electrochemical case. Therefore, if one sees a large number of charge degeneracy points [32] (more than the number of redox transitions that the molecule should be able to support stably) or comparatively low charging energies in a candidate SMT, one must be concerned that the device actually contains a metal nanoparticle. Some have argued that charging energies may be renormalized by strong coupling to the metallic source and drain [9]. Further, Coulomb charging effects can be strongly reduced in, for example, electrochemical STM experiments [33], due to the large relative dielectric constant of the surrounding solution.

Failing an independent imaging technique, the best diagnostic of all for SMTs is to measure a property that is intrinsic and specific to the molecule in question. Examples of such properties include Kondo conduction for known spin states of molecules, vibrational modes that agree with measured infrared and Raman spectra of molecules, optical response, etc.

\section{Experimental results}

Devices are measured in the dark in the variable temperature vacuum probe station, with a high surface area liquid nitrogen cold trap attached to the chamber to guarantee a low background pressure of potential condensates. Current-voltage characteristics are measured at dc using a semiconductor parameter analyzer. Numerical differentiation is used to compute both $\partial I_{\mathrm{D}} / \partial V_{\mathrm{SD}}$ and $\partial^{2} I_{\mathrm{D}} / \partial V_{\mathrm{SD}}^{2}$, and these results have been compared to those obtained directly from ac lock-in amplifier techniques with good agreement.

\subsection{Inelastic electron tunneling spectroscopy}

Fig. 4a shows a conductance map for a device incorporating the Co containing molecule at $5 \mathrm{~K}$. As with most of our devices, our gate coupling is sufficient to see one charge degeneracy point at $V_{\mathrm{G}} \sim+7 \mathrm{~V}$; that is, we can change the charge state of the molecule by one electron, with that charge compensated by the gate. A pronounced Kondo resonance is visible at zero source/drain bias in the right-hand charge state (tentatively $\mathrm{Co}(\mathrm{II})$ ), while traces of such a resonance are apparent in the left-hand charge state (tentatively $\mathrm{Co}(\mathrm{III}))$. We note that establishing strict correspondence between observed conduction and transition metal valence is complicated by the fact that the conjugated ligands may also be capable of changing their charge state. From the conductance map and the boundaries of the Coulomb blockade region, it is clear that the electron addition energy in this molecule exceeds $200 \mathrm{meV}$.

Further, note that there is asymmetry between positive and negative $V_{\mathrm{SD}}$. In such Coulomb blockade devices this kind of asymmetry is common, and has its origins in the asymmetric coupling between the island and the source and drain electrodes. In the limit of extreme asymmetry of coupling (e.g., in an STM experiment, where the tipmolecule tunneling coupling is much weaker than the molecule/substrate coupling), symmetric $I V$ curves can be restored, since the molecule essentially remains pinned energetically to the substrate. The lack of symmetric $I_{\mathrm{D}^{-}}$ $V_{\mathrm{SD}}$ curves about $V_{\mathrm{SD}}=0$ in these SMTs guarantees that vibrational features in $\partial^{2} I_{\mathrm{D}} / \partial V_{\mathrm{SD}}^{2}$ will not have symmetric intensities about $V_{\mathrm{SD}}=0$ in these structures. 

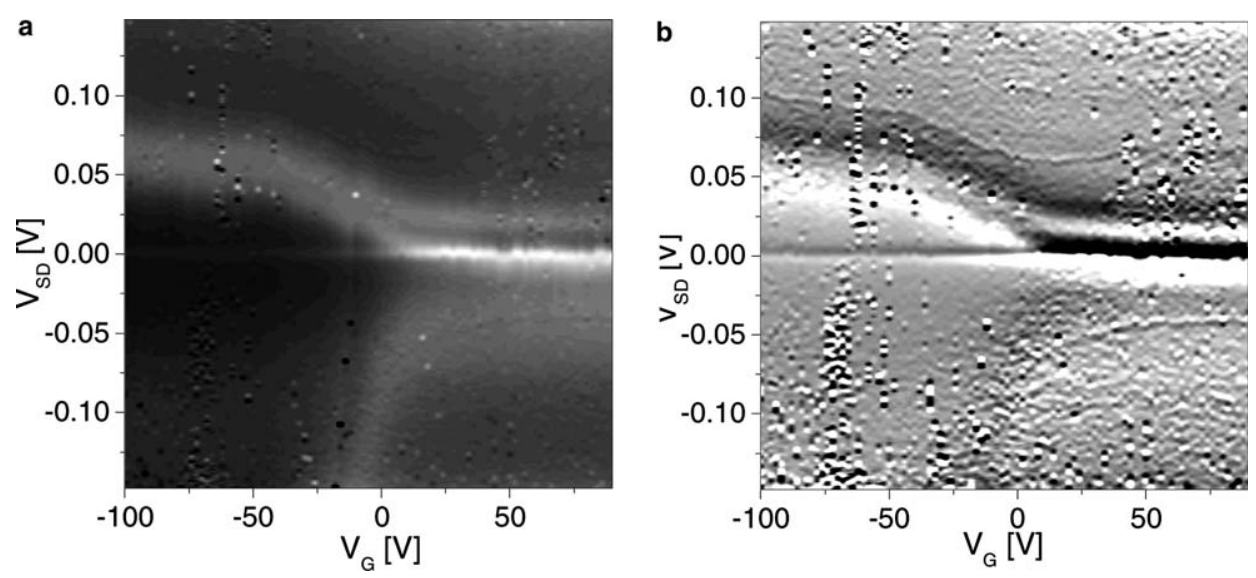

Fig. 4. Inelastic electron tunneling in a Co-complex SMT. (a) Map of differential conductance (black $=0$; white $=0.01 e^{2} / h$ ) at $5 \mathrm{~K}$ for this device, showing Coulomb blockade and transition to Kondo resonance in right-hand charge state. (b) Map of $\partial^{2} I_{\mathrm{D}} / \partial V_{\mathrm{SD}}^{2}$, showing narrow features at finite $V_{\mathrm{SD}}$ that correspond to inelastic electron tunneling via vibrational modes of the molecule.

Fig. $4 \mathrm{~b}$ shows $\partial^{2} I_{\mathrm{D}} / \partial V_{\mathrm{SD}}^{2}$ as a function of $V_{\mathrm{SD}}$ and $V_{\mathrm{G}}$, and two sharp features immediately stand out. One is at positive $V_{\mathrm{SD}}$ that follows the edges of the Coulomb blockade boundary at negative $V_{\mathrm{G}}$ and then curves into positive $V_{\mathrm{G}}$ territory before being lost in the noise. The other appears at negative $V_{\mathrm{SD}} \sim-50 \mathrm{mV}$ and positive $V_{\mathrm{G}}$. These features and others like them are seen with regularity in both the Coulomb blockaded regime, and as "satellites" of the Kondo resonance. In devices of apparently greater symmetry, where the $I_{\mathrm{D}}-V_{\mathrm{SD}}$ characteristics are more symmetric about $V_{\mathrm{SD}}=0$, a feature in $\partial^{2} I_{\mathrm{D}} / \partial V_{\mathrm{SD}}^{2}$ at positive bias is paired with an accompanying feature and negative bias, as in standard inelastic electron tunneling spectroscopy. The energies at which these features appear $\left(e V_{\mathrm{SD}}=E^{*}\right)$ are significantly smaller than the electron addition energies, and are therefore unlikely to be related to electronic excitations of the molecules.

From prior experience [12] we can identify these features as signatures of inelastic tunneling processes. When large numbers of devices are examined (over 400 electrode sets with molecules, total, with over 12 showing distinct inelastic cotunneling features), and the inelastic energies $E^{*}$ are histogrammed, good agreement is found between the locations of these inelastic peaks and features in the measured Raman and IR adsorption spectra of the molecules [12]. It is known theoretically that the "lineshapes" of these $\partial^{2} I_{\mathrm{D}} / \partial V_{\mathrm{SD}}^{2}$ features are nontrivial, and can either be peaks, dips, or Fano shapes depending on the microscopic details of the system. Similarly, the fact that each device exhibits only a small number of inelastic tunneling signatures is not surprising, since the relative intensity of such vibrational features can be altered dramatically by changes in single bonds near the junction [34].

The observation of such vibrational inelastic tunneling features is not surprising, since inelastic electron tunneling spectroscopy (IETS) of vibrational modes has been seen in tunnel junctions since the 1960s [35]. Indeed, IETS has undergone a recent resurgence with elegant single-molecule measurements made using STM [3], as well as measure- ments made on ensembles of molecules by crossed wire [36-38] and nanopore [39] methods.

The dependence of the vibrational energies on $V_{\mathrm{G}}$ is only observable with this SMT technique, and is the most interesting feature of the data. In the blockaded region or in the Kondo regime, far away from a charge degeneracy point, these features are independent of $V_{\mathrm{G}}$, as expected for standard inelastic cotunneling. However, near charge degeneracy, these vibrational modes can shift significantly. This is not necessarily surprising: a cation or anion can have shifted vibrational spectra relative to a neutral molecule, for example. Similarly, if the geometry of the molecule changes upon reduction or oxidation, one expects to see vibrational modes corresponding to displacements along the direction of the distortion soften as the redox state change is reached. Currently no theoretical treatment exists of this effect, which corresponds to an effective electronic control over the vibrational properties of individual molecules.

\subsection{The Kondo resonance and strong molecule-metal coupling}

As mentioned above, the Kondo resonance appears in systems with unpaired spins at temperatures below a characteristic Kondo temperature that is exponentially sensitive to the spin-leads coupling. Fig. 5 shows the conductance map of another Co-complex device with a prominent Kondo at zero bias in the right-hand charge state. The charge degeneracy point is at $V_{\mathrm{G}} \sim-30 \mathrm{~V}$. The full-width at half-maximum of the peak at $V_{\mathrm{G}}=+10 \mathrm{~V}$ is $13 \mathrm{mV}$. From the established correlation between Kondo temperature (determined by $T$ dependence of conductance peak height) and Kondo resonance width $[7,40,41]$, this corresponds to a Kondo temperature of $\sim 75 \mathrm{~K}$. Such strong, broad resonances have been seen in 40 out of over 1200 electrode sets examined containing transition metal complexes, with inferred Kondo temperatures ranging from 40 to $120 \mathrm{~K}$. 


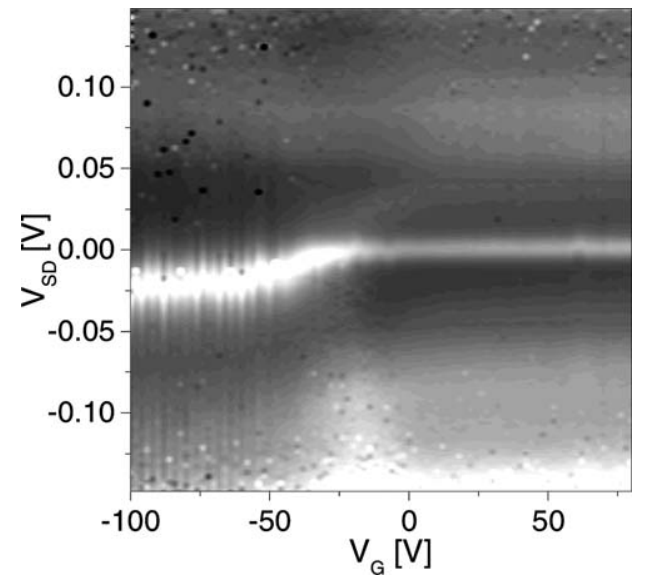

Fig. 5. Differential conductance map (black $=0$; white $=0.018 e^{2} / h$ ) of Co-complex SMT showing strong Kondo resonance in the right-hand charge state at $5 \mathrm{~K}$.

Weaker resonances (narrower, or with significantly lower temperatures of appearance) have not been observed in devices tested. Similarly, no Kondo resonances were observed in over 500 control junctions with no molecules, alkane chains, or other control molecules not containing unpaired spins. This is particularly important in light of recent work $[15,42,43]$ that demonstrates that it is possible to form metal nanoclusters on the molecular scale via electromigration, and that structures can show Kondo physics, albeit with much lower $(\sim 7 \mathrm{~K})$ Kondo temperatures [42]. We also note that such Kondo observations in all-metal devices are extremely sensitive to the details of the electromigration method, and that devices fabricated without the specific active feedback used in Ref. [42] do not show Kondo resonances [44]. The nontrivial electronic and optical properties of nanoclustered metal further underscore the importance of control experiments and a systematic approach to SMTs.

The Kondo effect has also been studied with STM by performing STS of single magnetic impurities on atomically smooth metal surfaces. Investigations of individual
Co atoms on $\mathrm{Au}(111)$ surfaces $[5,45]$ have found a Kondo temperature for that system of $\sim 70 \mathrm{~K}$. A detailed electronic structure study of charge transfer between the Co atom and the $\mathrm{Au}(111)$ surface combined with sophisticated manybody calculations of the Kondo correlations has successfully understood this result [46].

Remarkably, this similarity of Kondo temperatures implies that the spin degree of freedom in our transition metal complex is as strongly coupled to the delocalized $\mathrm{Au}$ conduction electrons as it would be if the Co were directly bonded metallically to the Au surface. This demonstrates conclusively that conjugated ligands of the type used in this complex can couple strongly to conduction electrons in a metal. Similar strong coupling has been reported recently in another conjugated system [47]. Detailed electronic structure calculations of the ligand/metal binding, with a realistic treatment of the Au surface, should help determine what makes these ligands particularly effective.

We note two very recent STM works $[48,49]$ have examined transition metal complexes physisorbed on noble metal surfaces. Both groups have observed extremely strong Kondo resonances with Kondo temperatures in some cases approaching $300 \mathrm{~K}$.

\subsection{Robust SMTs under thermal cycling}

We present one further new result with implications for the eventual development of technologies based on SMTs. Previous investigations of SMTs have been performed at cryogenic temperatures in part because device geometric stability at the atomic level is difficult to maintain at room temperature in such systems. In the low temperature electromigration approach to SMT fabrication, the structure of the resulting electrodes and interfaces is frozen in a metastable state. Upon warming the devices to room temperature, annealing, surface reconstruction, and surface diffusion of $\mathrm{Au}$ atoms can significantly modify device conductance irreversibly, by altering the interelectrode geometry.

Fig. 6 shows the conductance map of a particular Cocomplex SMT at $5 \mathrm{~K}$, on three successive days. Between
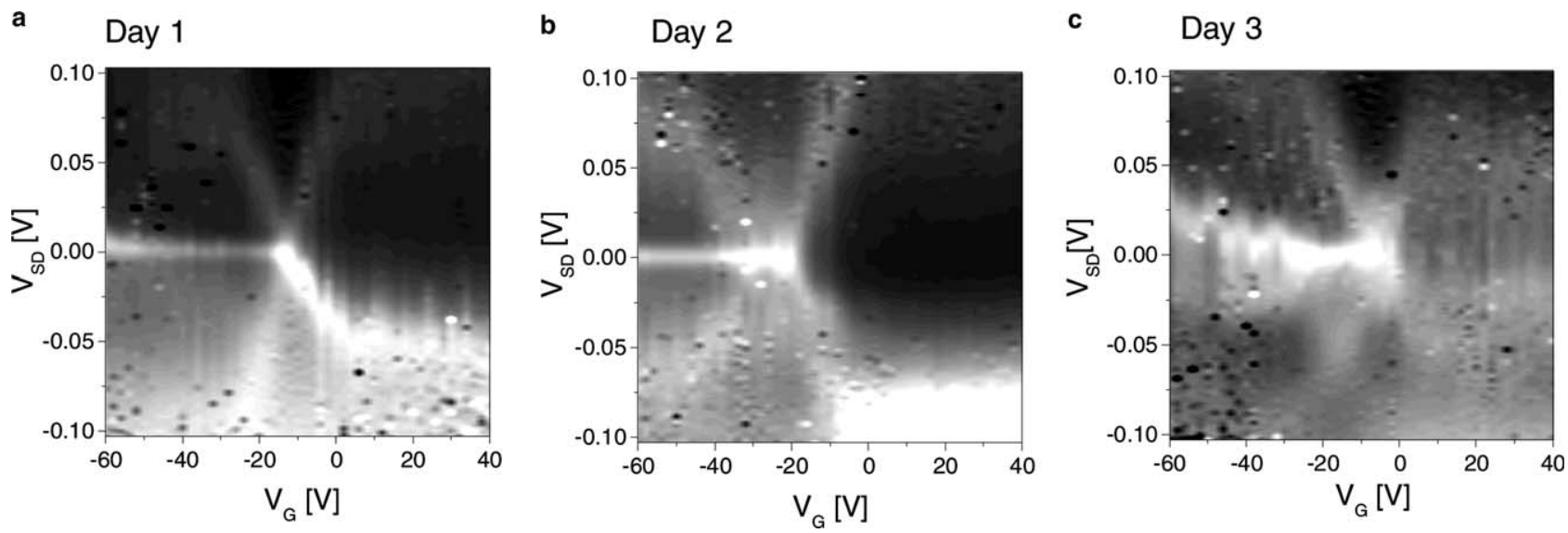

Fig. 6. Differential conductance map (black $=0$; white $=0.76 e^{2} / h$ ) of Co-complex SMT at $5 \mathrm{~K}$ on three successive days. Between each day, the device spent at least $18 \mathrm{~h}$ at $300 \mathrm{~K}$. 
(a) and (b), the sample was warmed (in high vacuum) to room temperature and allowed to sit for $18 \mathrm{~h}$ before being re-cooled. A similar thermal cycling took place between (b) and (c). While details of the map change, the main essentials remain: a well-defined Coulomb blockade with electron addition energy in excess of $100 \mathrm{meV}$, with a charge degeneracy point between $V_{\mathrm{G}}=-20$ and $0 \mathrm{~V}$.

This particular device is clearly relatively robust under extended times at $300 \mathrm{~K}$. Indeed, this device is substantially more stable than molecule-free metal junctions, the conductance of which can change by orders of magnitude on hour time scales due to surface diffusion of $\mathrm{Au}$ atoms. We believe, though cannot verify directly, that the strong covalent bonding of the molecule to both source and drain electrodes is one reason for this impressive stability relative to bare junctions. By choosing a more refractory metal for electrodes, and with improvements in surface preparation, we believe that SMTs capable of room temperature operation are possible.

\section{Conclusions}

We have given an overview of single-molecule transistor fabrication, characterization, and operation, and a discussion of the relevant chemical synthesis and surface chemistry. We present three recent results: SMTs used for gate-modulated inelastic tunneling spectroscopy of single molecules; the Kondo resonance in SMTs as a demonstration that conjugated ligands can provide very strong coupling of molecular spin degrees of freedom to underlying metal conduction electrons; and data showing that with appropriate surface chemistry SMTs can be sufficiently robust to survive extended periods at room temperature.

A number of practical issues remain before SMTs may be utilized to their full potential. There is much room for improvement in current fabrication and characterization techniques. While the electromigration approach does produce enough devices with sufficient reproducibility for academic study, it is hard to believe that there is not a better possible procedure that would raise the yield of devices to greater than the current ceiling of $10-20 \%$. Diagnostic tools independent from electronic transport measurements must be developed that will lend SMTs the same degree of reproducible control as STM investigations.

Scientifically, there also remain a large number of open issues. Every electronic transport measurement in such a device is inherently a highly nonequilibrium experiment, with electronic distribution functions in the leads remaining nonthermal over distances much larger than the scale of the molecule. Relaxational couplings between molecular vibrations and bulk phonons of the leads are not known, while electronic transport can pump vibrational modes, resulting in extremely athermal vibrational distributions. Single physisorbed or chemisorbed impurities or charged defects [50] can profoundly shift local charge transfer and energetics in manners which are challenging to control or predict from first principles. Virtually all theoretical treat- ments of electronic conduction through SMTs neglect electronic correlation effects such as Kondo exchange, while such physics can profoundly affect transport.

In the brief time since their inception, SMTs have demonstrated themselves to be excellent and unique tools for studying physics and physical chemistry problems on a previously inaccessible scale. With the development of these novel nanostructures, and the enormous capabilities available from the chemistry community, the next years promise to be an exciting time for molecular electronics at the single-molecule level.

\section{Acknowledgments}

D.N. acknowledges the financial support from the Research Corporation, the Robert A. Welch Foundation, the David and Lucille Packard Foundation, an Alfred P. Sloan Foundation Fellowship, and NSF CAREER award DMR-0347253. J.M.T. acknowledges the support from DARPA via the AFOSR.

\section{Note added in proof}

The mechanism of the thiocyanate assembly has been discussed in two publications: [51,52].

\section{References}

[1] X.H. Lu, M. Grobis, K.H. Khoo, S.G. Louie, M.F. Crommie, Phys. Rev. B 70 (2004) 115418.

[2] X.H. Qiu, G.V. Nazin, W. Ho, Phys. Rev. Lett. 92 (2004) 206102.

[3] B.C. Stipe, M.A. Rezaei, W. Ho, Science 280 (1998) 1732.

[4] A.J. Heinrich, J.A. Gupta, C.P. Lutz, D.M. Eigler, Science 306 (2004) 466.

[5] V. Madhavan, W. Chen, T. Jamneala, M.F. Crommie, N.S Wingreen, Science 280 (1998) 567.

[6] H. Park, J. Park, A.K.L. Lim, E.H. Anderson, A.P. Alivisatos, P.L. McEuen, Nature 407 (2000) 57.

[7] J. Park, A.N. Pasupathy, J.I. Goldsmith, C. Chang, Y. Yaish, J.R. Petta, M. Rinkowski, J.P. Sethna, H.D. Abruña, P.L. McEuen, D.C. Ralph, Nature 417 (2002) 722.

[8] W. Liang, M.P. Shores, M. Bockrath, J.R. Long, H. Park, Nature 417 (2002) 725

[9] S. Kubatkin, A. Danilov, M. Hjort, J. Cornil, J.-L. Brèdas, N. StuhrHansen, P. Hedegård, T. Bjørnholm, Nature 425 (2003) 698.

[10] J. Park, A.N. Pasupathy, J.I. Goldsmith, A.V. Soldatov, C. Chang, Y. Yaish, J.P. Sethna, H. Abruña, D.C. Ralph, P.L. McEuen, Thin Solid Films 438-439 (2003) 457.

[11] L.H. Yu, D. Natelson, Nanotechnology 15 (2004).

[12] L.H. Yu, Z.K. Keane, J.W. Ciszek, L. Cheng, M.P. Stewart, J.M. Tour, D. Natelson, Phys. Rev. Lett. 93 (2004) 266802.

[13] A.N. Pasupathy, R.C. Bialczak, J. Martinek, J.E. Grose, L.A.K. Donev, P.L. McEuen, D.C. Ralph, Science 306 (2004) 86.

[14] H. Park, A.K.L. Lim, A.P. Alivisatos, J. Park, P.L. McEuen, Appl. Phys. Lett. 75 (1999) 301

[15] T.H. Lee, J.I. Gonzalez, R.M. Dickson, Proc. Natl. Acad. Sci. USA 99 (2002) 10272.

[16] Single Charge Tunneling: Coulomb Blockade Phenomena in Nanostructures, Plenum Press, New York, 1992.

[17] D.L. Klein, R. Roth, A.K.L. Lim, A.P. Alivisatos, P.L. McEuen, Nature 389 (1997) 699.

[18] H.M. McConnell, J. Chem. Phys. 35 (1961) 508. 
[19] D.V. Averin, Y.V. Nazarov, in: H.G.a.M.H. Devoret (Ed.), Single Charge Tunneling: Coulomb Blockade Phenomena in Nanostructures, Plenum Press, New York, 1992, p. 217.

[20] S. De Franceschi, S. Sasaki, J.M. Elzerman, W.G. van der Wiel, S. Tarucha, L.P. Kouwenhoven, Phys. Rev. Lett. 86 (2001) 878.

[21] W.J. Liang, M. Bockrath, H. Park, Phys. Rev. Lett. 88 (2002) 126801.

[22] A. Troisi, M.A. Ratner, A. Nitzan, J. Chem. Phys. 118 (2003) 6072.

[23] M. Galperin, M.A. Ratner, A. Nitzan, J. Chem. Phys. 121 (2004) 11965.

[24] A.T. Johnson, L.P. Kouwenhoven, W. Dejong, N.C. Vandervaart, C.J.P.M. Harmans, C.T. Foxon, Phys. Rev. Lett. 69 (1992) 1592.

[25] D.R. Stewart, D. Sprinzak, C.M. Marcus, C.I. Duruoz, J.S. Harris, Science 278 (1997) 1784

[26] L.P. Kouwenhoven, T.H. Oosterkamp, M.W.S. Danoesastro, M. Eto, D.G. Austing, T. Honda, S. Tarucha, Science 278 (1997) 1788.

[27] J. Kondo, Prog. Theor. Phys. 32 (1964) 37.

[28] F.D.M. Haldane, Phys. Rev. Lett. 40 (1978) 416.

[29] D. Goldhaber-Gordon, J. Gores, M.A. Kastner, H. Shtrikman, D. Mahalu, U. Meirav, Phys. Rev. Lett. 81 (1998) 5225.

[30] D. Goldhaber-Gordon, H. Shtrikman, D. Mahalu, D. AbuschMagder, U. Meirav, M.A. Kastner, Nature 391 (1998) 156.

[31] S.M. Cronenwett, T.H. Oosterkamp, L.P. Kouwenhoven, Nature 281 (1998) 540.

[32] H. Yu, Y. Luo, K. Beverly, J.F. Stoddart, H.-R. Tseng, J.R. Heath, Angew. Chem. Int. Ed. 42 (2003) 5706.

[33] X. Xiao, L.A. Nagahara, A.M. Rawlett, N.J. Tao, J. Am. Chem. Soc. 127 (2005) 9235.

[34] Y.-C. Chen, M. Zwolak, M.D. Ventra, Nanoletters 4 (2004) 1709.

[35] R.C. Jaklevic, J. Lambe, Phys. Rev. Lett. 17 (1966) 1139.

[36] S. Gregory, Phys. Rev. Lett. 64 (1990) 689.
[37] D.T. Zimmerman, G. Agnolet, Rev. Sci. Instrum. 72 (2001) 1781.

[38] J.G. Kushmerick, J. Lazorcik, C.H. Patterson, R. Shashidhar, D.S. Seferos, G.C. Bazan, Nanoletters 4 (2004) 639.

[39] W.Y. Wang, T. Lee, I. Kretzschmar, M.A. Reed, Nanoletters 4 (2004) 643.

[40] K. Nagaoka, T. Jamneala, M. Grobis, M.F. Crommie, Phys. Rev. Lett. 88 (2002).

[41] L.H. Yu, Z.K. Keane, J.W. Ciszek, J.M. Tour, T. Baruah, M.R. Pederson, D. Natelson, Phys. Rev. Lett. 95 (2005), in press.

[42] A.A. Houck, J. Labaziewicz, E.K. Chan, J.A. Folk, I.L. Chuang, Nanoletters 5 (2005)

[43] J.I. Gonzalez, T.H. Lee, M.D. Barnes, Y. Antoku, R.M. Dickson, Phys. Rev. Lett. 93 (2004) 147402.

[44] J.A. Folk, personal communication, 2005.

[45] V. Madhavan, W. Chen, T. Jamneala, M.F. Crommie, N.S. Wingreen, Phys. Rev. B 64 (2001) 165412.

[46] O. Ujsaghy, J. Kroha, L. Szunyogh, A. Zawadowski, Phys. Rev. Lett. $85(2000) 2557$.

[47] S.A. Getty, C. Engtrakul, L. Wang, R. Liu, S.-H. Ke, H.U. Baranger, W. Yang, M.S. Fuhrer, L.R. Sita, Phys. Rev. B 71 (2005) 241401.

[48] P. Wahl, L. Diekhöner, G. Wittich, L. Vitali, M.A. Schneider, K. Kern, Phys. Rev. Lett. 95 (2005) 166601.

[49] A. Zhao, Q. Li, L. Chan, H. Xiang, W. Wang, S. Pan, B. Wang, X. Xiao, J. Yang, J.G. Hou, Q. Zhu, Science 309 (2005) 1542.

[50] P.G. Piva, G.A. DiLabio, J.L. Pitters, J. Zikovsky, M. Rezeq, S. Dogel, W. Hofer, R.A. Wolkow, Nature 435 (2005) 658.

[51] J.W. Ciszek, M.P. Stewart, J.M. Tour, J. Am. Chem. Soc. 126 (2004) 13172.

[52] J.W. Ciszek, J.M. Tour, Chem. Mater. 17 (2005) 5684. 